PROCEEDINGS OF THE

AMERICAN MATHEMATICAL SOCIETY

Volume 138, Number 7, July 2010, Pages 2463-2475

S 0002-9939(10)10253-6

Article electronically published on February 24, 2010

\title{
THE DEFECT INDEX OF SINGULAR SYMMETRIC LINEAR DIFFERENCE EQUATIONS WITH REAL COEFFICIENTS
}

\author{
GUOJING REN AND YUMING SHI
}

(Communicated by Nigel J. Kalton)

\begin{abstract}
This paper is concerned with the defect index of singular symmetric linear difference equations of order $2 n$ with real coefficients and one singular endpoint. We show that their defect index $d$ satisfies the inequalities $n \leq d \leq 2 n$ and that all values of $d$ in this range are realized. This parallels the well known result of Glazman for differential equations established about 1950. In addition, several criteria of the limit point and strong limit point cases are established.
\end{abstract}

\section{INTRODUCTION}

In this paper we are interested in the defect index of the following singular symmetric linear difference equation with real coefficients:

$$
\sum_{j=0}^{n}(-1)^{j} \Delta^{j}\left[p_{j}(t) \nabla^{j} y(t)\right]=\lambda w(t) y(t), \quad t \in I,
$$

where $\Delta$ and $\nabla$ are forward and backward difference operators, respectively; i.e., $\Delta y(t)=y(t+1)-y(t)$ and $\nabla y(t)=y(t)-y(t-1) ; I:=[0,+\infty)=\{t\}_{t=0}^{+\infty} ; w(t)>0$ and $p_{n}(t) \neq 0$ for $t \in I$; and $\lambda$ is a complex spectral parameter.

By letting $u(t)=\left(u_{1}(t), u_{2}(t), \ldots, u_{2 n}(t)\right)^{T}$ with

$$
u_{j}(t)=\Delta^{j-1} y(t-j), \quad u_{n+j}(t)=\sum_{k=j}^{n}(-1)^{k-j} \Delta^{k-j}\left(p_{k}(t) \nabla^{k} y(t)\right)
$$

for $1 \leq j \leq n$, equation (1.1) can be converted into the following singular linear discrete Hamiltonian system

$$
J \Delta u(t)=(\lambda W(t)+P(t)) R(u)(t), \quad t \in I,
$$

where

$$
W(t)=\operatorname{diag}\{w(t), 0, \ldots, 0\}, \quad P(t)=\left(\begin{array}{cc}
-C(t) & A^{*}(t) \\
A(t) & B(t)
\end{array}\right)
$$

Received by the editors July 6, 2009, and, in revised form, October 9, 2009.

2010 Mathematics Subject Classification. Primary 39A70, 34B20.

Key words and phrases. Singular symmetric linear difference equation, square summable solution, defect index, limit point case, strong limit point case.

This research was supported by the NNSF of Shandong Province (Grant Y2006A15).

(C)2010 American Mathematical Society Reverts to public domain 28 years from publication 
with

$$
\begin{aligned}
& A(t)=\left(\begin{array}{cc}
0 & I_{n-1} \\
0 & 0
\end{array}\right), \quad B(t)=\operatorname{diag}\left\{0, \ldots, 0, p_{n}^{-1}(t)\right\}, \\
& C(t)=\operatorname{diag}\left\{p_{0}(t), p_{1}(t), \ldots, p_{n-1}(t)\right\}
\end{aligned}
$$

$I_{n-1}$ is the $(n-1) \times(n-1)$ unit matrix, and the right partial shift operator

$$
R(u)(t)=\left(u_{1}(t+1), \ldots, u_{n}(t+1), u_{n+1}(t), \ldots, u_{2 n}(t)\right)^{T} .
$$

According to the classical von Neumann theory (cf. [11, 15]) and its generalization [5], a symmetric operator or a non-densely defined Hermitian operator has a self-adjoint extension if and only if its positive and negative defect indices are equal and its self-adjoint extension domains have a close relationship with its defect index. So it is very important to determine the defect indices of both differential equations and difference equations in the study of self-adjoint extensions for differential and difference equations.

The problem on the defect index $d$ of the singular symmetric linear differential equation with real coefficients

$$
\sum_{j=0}^{n}(-1)^{j}\left[p_{j}(x) y^{(j)}(x)\right]^{(j)}=\lambda w(x) y(x), \quad x \in(a, b),
$$

has been studied for a long time. It is well known that $d$ is equal to the number of linearly independent square integrable solutions of (1.6) for each $\lambda \in \mathbb{C} \backslash \mathbb{R}$, where $\mathbb{C}$ and $\mathbb{R}$ denote the sets of the complex and real numbers, respectively. In particular, I. M. Glazman [8] showed that the defect index $d$ of equation (1.6) defined on $(0,+\infty)$, where $x=0$ is a regular endpoint, satisfied the inequalities $n \leq d \leq 2 n$ and all values of $d$ in this range. Many other results on the defect index $d$ of equation (1.6) were summarized in [7] and [11, Chapter V].

For the discrete case, F. V. Atkinson first studied the number of linearly independent square summable solutions of second-order symmetric linear difference equations [1. Subsequently, his work was further developed (cf. [2, 3, 9, 10, [12-114). Recently, some Weyl-Titchmarsh fundamental theory of singular linear discrete Hamiltonian systems was established [4, 12. In the present paper, we study the defect index of equation (1.1) by applying the Weyl-Titchmarsh fundamental theory of singular linear discrete Hamiltonian systems and asymptotic behaviors of solutions of perturbed difference equations.

The rest of the paper is organized as follows. In Section 2, two equivalent forms of equation (1.1) are formulated, and a result on asymptotic behaviors of solutions of difference equations and some useful lemmas are stated. In Section 3, we pay attention to the defect index of equation (1.1) and its equivalent forms. Theorem 3.1 and Example 3.2 show that the defect index $d$ of equation (1.1) satisfies the inequalities $n \leq d \leq 2 n$ and all values of $d$ in this range are realized. This parallels the above Glazman's result for differential equations. In addition, several criteria of the limit point and strong limit point cases are established.

\section{Preliminaries}

This section is divided into four subsections. In Subsections 2.1 and 2.2, a classification of limit cases of equation (1.1) and two equivalent forms of equation (1.1) are introduced, respectively. In Subsection 2.3, some sufficient and necessary 
conditions of the limit point case and the strong limit point case of equation (1.1) are given. Finally, in Subsection 2.4, asymptotic behaviors of solutions of an equivalent form to equation (1.1) are studied.

2.1. Classification of limit cases. By $\mathbb{C}$ denote the set of the complex numbers, and by $\bar{z}$ and $u^{*}$ denote the conjugate of $z$ and the complex conjugate transpose of $u$, respectively.

We now introduce the space

$$
l_{w}^{2}[0,+\infty):=\left\{y=\{y(t)\}_{t=-n}^{+\infty} \subset \mathbb{C}: \sum_{t=0}^{+\infty} w(t)|y(t)|^{2}<+\infty\right\}
$$

with inner product

$$
\langle x, y\rangle_{w}:=\sum_{t=0}^{+\infty} w(t) \bar{y}(t) x(t)
$$

where the weight function $w(t)>0$ on $I$. For $x, y \in l_{w}^{2}[0,+\infty), x$ is said to be equal to $y$ if $\|x-y\|_{w}=0$, where $\|\cdot\|_{w}:=\left(\langle\cdot, \cdot\rangle_{w}\right)^{1 / 2}$. In this sense, $l_{w}^{2}[0,+\infty)$ is a Hilbert space with the inner product $\langle\cdot, \cdot\rangle_{w}$. In the special case of $w(t) \equiv 1$, $l_{w}^{2}[0,+\infty)$ is briefly denoted by $l^{2}[0,+\infty)$.

Similarly to the scalar case, denote

$$
L_{W}^{2}[0,+\infty):=\left\{u=\{u(t)\}_{t=0}^{+\infty} \subset \mathbb{C}^{2 n}: \sum_{t=0}^{+\infty} R^{*}(u)(t) W(t) R(u)(t)<+\infty\right\}
$$

with the inner product

$$
\langle u, v\rangle_{W}:=\sum_{t=0}^{+\infty} R^{*}(v)(t) W(t) R(u)(t),
$$

where the weight function $W(t)$ is a $2 n \times 2 n$ non-negative Hermitian matrix and $R(\cdot)$ is defined by $(1.5)$. Then $L_{W}^{2}[0,+\infty)$ is a Hilbert space with the inner product $\langle\cdot, \cdot\rangle_{W}$ in the sense that $u=v$ if $\|u-v\|_{W}=0$, where $\|\cdot\|_{W}:=\left(\langle\cdot, \cdot\rangle_{W}\right)^{1 / 2}$.

As stated in the previous section, (1.1) can be converted into (1.3) by (1.2). It is evident that for any $\lambda \in \mathbb{C}$, if $y(t)$ is a solution of (1.1), then $u(t)$ defined by (1.2) is a solution of (1.3) and conversely, if $u(t)$ is a solution of (1.3), then $y(t)=u_{1}(t+1)$ is a solution of (1.1). Moreover, it follows from (1.2) and (1.4) that

$$
\|u\|_{W}^{2}=\sum_{t=0}^{+\infty} R^{*}(u)(t) W(t) R(u)(t)=\sum_{t=0}^{+\infty} w(t)|y(t)|^{2}=\|y\|_{w}^{2} .
$$

By $d_{w}(\lambda)$ and $D_{W}(\lambda)$ denote the number of the linearly independent solutions of equation (1.1) in $l_{w}^{2}[0,+\infty)$ and system $(1.3)$ in $L_{W}^{2}[0,+\infty)$, respectively. Then the following result is obtained:

Lemma 2.1. $D_{W}(\lambda)=d_{w}(\lambda)$ for any $\lambda \in \mathbb{C}$.

Denote $d_{+}=d_{w}(i)$ and $d_{-}=d_{w}(-i)$. By [12, Theorem 5.1], $d_{+}$and $d_{-}$are equal to the positive and negative defect indices of the minimal operator generated by (1.1), respectively. The following result is directly derived from Corollary 4.1 and Theorem 5.4 in [12] and Lemma 2.1:

Lemma 2.2. $d_{w}(\lambda)=d_{+}=d_{-}$is a constant for all $\lambda \in \mathbb{C}$ with $\operatorname{Im} \lambda \neq 0$, and $n \leq d \leq 2 n$. 
Definition 2.3. The constant $d:=d_{ \pm}$is called the defect index of equation (1.1), and equation (1.1) is said to be in the limit- $d$ case at $t=+\infty$. In the special case $d=n,(1.1)$ is said to be in the limit point case (l.p.c.) at $t=+\infty$, and in the other special case $d=2 n,(1.1)$ is said to be in the limit circle case (l.c.c.) at $t=+\infty$.

2.2. Two equivalent forms of equation (1.1). In this subsection we formulate two equivalent forms of (1.1), one of which has a weight function being equivalent to 1 and the other has a leading-term coefficient being equivalent to $(-1)^{n}$. For convenience, we first introduce the following useful formulae:

$$
\begin{aligned}
& \Delta^{k} y(t)=\nabla^{k} y(t+k)=\sum_{j=0}^{k}(-1)^{k-j} C_{k}^{j} y(t+j), \\
& \Delta^{k}(a(t) b(t))=\sum_{j=0}^{k} C_{k}^{j} \Delta^{j} a(t) \Delta^{k-j} b(t+j),
\end{aligned}
$$

where $C_{k}^{j}=k ! /(j !(k-j) !)$ is the binomial coefficient. By using (2.1), we have from (1.1) that

$$
\sum_{j=0}^{n} P_{j}(t+j) y(t+j)+\sum_{j=1}^{n} P_{j}(t) y(t-j)=\lambda w(t) y(t), \quad t \in I,
$$

where

$$
P_{j}(t)=(-1)^{j} \sum_{s=j}^{n} \sum_{k=0}^{s-j} C_{s}^{k} C_{s}^{s-j-k} p_{s}(t+k), \quad 0 \leq j \leq n,
$$

or

$$
\begin{aligned}
& p_{n}(t)=(-1)^{n} P_{n}(t), \\
& p_{j}(t)=(-1)^{j} P_{j}(t)-\sum_{s=j+1}^{n} \sum_{k=0}^{s-j} C_{s}^{k} C_{s}^{s-j-k} p_{s}(t+k), 0 \leq j \leq n-1 .
\end{aligned}
$$

First, setting

$$
y(t)=w^{-1 / 2}(t) x(t)
$$

and multiplying $w^{-1 / 2}(t)$ on both sides of (2.3), we get

$$
\sum_{j=0}^{n} Q_{j}(t+j) x(t+j)+\sum_{j=1}^{n} Q_{j}(t) x(t-j)=\lambda x(t), \quad t \in I,
$$

where

$$
Q_{j}(t)=P_{j}(t) w^{-1 / 2}(t) w^{-1 / 2}(t-j), \quad 0 \leq j \leq n .
$$

Further, (2.7) can be rewritten as

$$
\sum_{j=0}^{n}(-1)^{j} \Delta^{j}\left[q_{j}(t) \nabla^{j} x(t)\right]=\lambda x(t), \quad t \in I,
$$

where $q_{j}(t)$ are determined by (2.5) with $P_{j}(t)$ and $p_{s}(t)$ replaced by $Q_{j}(t)$ and $q_{s}(t)$, respectively. It is evident that $q_{j}(t), 0 \leq j \leq n$, are all real-valued and $q_{n}(t) \neq 0$ for all $t \in I$. 
Second, setting $y(t)=g(t) z(t)$ and multiplying $g(t)$ on both sides of (2.3), where $g(t)$ is defined by

$$
g(-1)=g(-2)=\cdots=g(-n)=1, \quad g(j)=\frac{(-1)^{n}}{p_{n}(j)}, \quad 0 \leq j \leq n-1,
$$

and

$$
g(t)=\left\{\begin{array}{l}
\frac{p_{n}(t-n) p_{n}(t-3 n) \cdots p_{n}(j-n)}{p_{n}(t) p_{n}(t-2 n) \cdots p_{n}(j)}, \quad t=2 n k+j, k \geq 0, n \leq j \leq 2 n-1, \\
\frac{(-1)^{n} p_{n}(t-n) p_{n}(t-3 n) \cdots p_{n}(j+n)}{p_{n}(t) p_{n}(t-2 n) \cdots p_{n}(j)}, t=2 n k+j, k \geq 1,0 \leq j \leq n-1,
\end{array}\right.
$$

we get

$$
\sum_{j=0}^{n} R_{j}(t+j) z(t+j)+\sum_{j=1}^{n} R_{j}(t) z(t-j)=\lambda w_{0}(t) z(t), \quad t \in I,
$$

where

$$
\begin{aligned}
& R_{n}(t)=(-1)^{n}, \quad R_{j}(t)=P_{j}(t) g(t) g(t-j), \quad 0 \leq j \leq n-1, \\
& w_{0}(t)=w(t) g^{2}(t) .
\end{aligned}
$$

Further, (2.10) can be rewritten as

$$
(-1)^{n} \Delta^{n} \nabla^{n} z(t)+\sum_{j=0}^{n-1}(-1)^{j} \Delta^{j}\left[r_{j}(t) \nabla^{j} z(t)\right]=\lambda w_{0}(t) z(t), \quad t \in I,
$$

where $r_{j}(t)$ are determined by (2.5) with $P_{j}(t)$ and $p_{s}(t)$ replaced by $R_{j}(t)$ and $r_{s}(t)$, respectively. In this case, $r_{j}(t), 0 \leq j \leq n-1$, are all real-valued and $w_{0}(t)>0$ for $t \in I$.

It is evident that for any $\lambda \in \mathbb{C}, y(t)$ is a solution of equation (1.1) if and only if $x(t)=w^{1 / 2}(t) y(t)$ is a solution of equation (2.9) and if and only if $z(t)=g^{-1}(t) y(t)$ is a solution of equation (2.11). Moreover, it follows that

$$
\sum_{t=0}^{+\infty} w(t)|y(t)|^{2}=\sum_{t=0}^{+\infty}|x(t)|^{2}=\sum_{t=0}^{+\infty} w_{0}(t)|z(t)|^{2}
$$

By $d(\lambda)$ and $d_{w_{0}}(\lambda)$ denote the numbers of the linearly independent solutions of equation (2.9) in $l^{2}[0,+\infty)$ and of equation (2.11) in $l_{w_{0}}^{2}[0,+\infty)$, respectively. Then we have by $(2.12)$ and Lemma 2.2 that

$$
d_{w_{0}}(\lambda)=d(\lambda)=d_{w}(\lambda) \equiv d, \quad \operatorname{Im} \lambda \neq 0 .
$$

This means that equation (1.1) has the same defect index as those of its equivalent forms (2.9) and (2.11).

2.3. Sufficient and necessary conditions of the limit point case and the strong limit point case. The natural difference operator corresponding to equation (1.1) is defined by

$$
\mathcal{L}(y)(t):=\sum_{j=0}^{n}(-1)^{j} \Delta^{j}\left[p_{j}(t) \nabla^{j} y(t)\right], \quad t \in I,
$$


and the bilinear form $[\cdot, \cdot]$ associated with $(1.1)$ is defined by

$$
\begin{aligned}
{[x, y](t):=} & \sum_{j=1}^{n}\left(\sum_{k=j}^{n}(-1)^{k-j} \Delta^{k-j}\left[p_{k}(t) \nabla^{k} \bar{y}(t)\right]\right) \Delta^{j-1} x(t-j) \\
& -\sum_{j=1}^{n} \Delta^{j-1} \bar{y}(t-j)\left(\sum_{k=j}^{n}(-1)^{k-j} \Delta^{k-j}\left[p_{k}(t) \nabla^{k} x(t)\right]\right) .
\end{aligned}
$$

Then, for any $x(t)$ and $y(t)$ defined in $\{t\}_{t=-n}^{+\infty}$ we have

$$
\sum_{t=0}^{m}[\bar{y}(t)(\mathcal{L} x)(t)-(\overline{\mathcal{L} y})(t) x(t)]=\left.[x, y](t)\right|_{t=0} ^{m+1} .
$$

Denote $\mathcal{D}:=\left\{y \in l_{w}^{2}[0,+\infty): w^{-1} \mathcal{L} y \in l_{w}^{2}[0,+\infty)\right\}$, which is the domain of the maximal operator corresponding to operator $\mathcal{L}$. This yields from $(2.13)$ that $\lim _{t \rightarrow+\infty}[x, y](t)=c$ for any $x, y \in \mathcal{D}$, where $c$ is a finite constant. The following lemma is a direct consequence of [12, Theorem 6.15].

Lemma 2.4. Equation (1.1) is in l.p.c. at $t=+\infty$ if and only if for all $x, y \in \mathcal{D}$,

$$
\lim _{t \rightarrow+\infty}[x, y](t)=0 .
$$

Lemma 2.4 gives a sufficient and necessary condition of the limit point case for equation (1.1). In [14, Definition 2.1], a concept of the strong limit point case is introduced for a singular discrete Hamiltonian system. Following their definition, we give the following concept:

Definition 2.5. Equation (1.1) is said to be in the strong limit point case (s.l.p.c.) at $t=+\infty$ if for all $x, y \in \mathcal{D}$,

$$
\lim _{t \rightarrow+\infty} \sum_{j=1}^{n} \Delta^{j-1} \bar{x}(t-j)\left(\sum_{k=j}^{n}(-1)^{k-j} \Delta^{k-j}\left[p_{k}(t) \nabla^{k} y(t)\right]\right)=0 .
$$

For convenience, denote

$$
S(y)(t):=\sum_{j=1}^{n} \Delta^{j-1} \bar{y}(t-j)\left(\sum_{k=j}^{n}(-1)^{k-j} \Delta^{k-j}\left[p_{k}(t) \nabla^{k} y(t)\right]\right) .
$$

The following result is a sufficient and necessary condition of the strong limit point case for equation (1.1), which is a direct consequence of [14, Theorem 2.1].

Lemma 2.6. Equation (1.1) is in s.l.p.c. at $t=+\infty$ if and only if $\lim _{t \rightarrow+\infty} S(y)(t)$ $=0$ for all $y \in \mathcal{D}$.

Lemma 2.7. Assume that there exist a constant $c$ and an integer $t_{0} \geq 0$ such that $p_{j}(t)(1 \leq j \leq n)$ and $p_{0}(t)-c w(t)$ are either non-negative for all $t \geq t_{0}$ or non-positive for all $t \geq t_{0}$. Then $\lim _{t \rightarrow+\infty} S(y)(t)$ exists, finite or infinite, for all $y \in \mathcal{D}$. 
Proof. Using the first relationship of (2.1) and (2.2), we have

$$
\begin{aligned}
\Delta S(y)(t)= & \sum_{j=1}^{n} \Delta^{j} \bar{y}(t-j)\left(\sum_{k=j}^{n}(-1)^{k-j} \Delta^{k-j}\left[p_{k}(t) \nabla^{k} y(t)\right]\right) \\
& +\sum_{j=1}^{n} \Delta^{j-1} \bar{y}(t-j+1)\left(\sum_{k=j}^{n}(-1)^{k-j} \Delta^{k-j+1}\left[p_{k}(t) \nabla^{k} y(t)\right]\right) \\
= & \sum_{j=1}^{n} \Delta^{j} \bar{y}(t-j)\left(\sum_{k=j}^{n}(-1)^{k-j} \Delta^{k-j}\left[p_{k}(t) \nabla^{k} y(t)\right]\right) \\
& -\sum_{j=0}^{n-1} \Delta^{j} \bar{y}(t-j)\left(\sum_{k=j+1}^{n}(-1)^{k-j} \Delta^{k-j}\left[p_{k}(t) \nabla^{k} y(t)\right]\right) \\
= & \sum_{j=1}^{n} p_{j}(t)\left|\Delta^{j} y(t-j)\right|^{2}+\bar{y}(t)\left(\sum_{k=1}^{n}(-1)^{k+1} \Delta^{k}\left[p_{k}(t) \nabla^{k} y(t)\right]\right) \\
= & \sum_{j=1}^{n} p_{j}(t)\left|\Delta^{j} y(t-j)\right|^{2}+\bar{y}(t)\left(p_{0}(t) y(t)-(\mathcal{L} y)(t)\right) \\
= & \sum_{j=0}^{n} p_{j}(t)\left|\Delta^{j} y(t-j)\right|^{2}-\bar{y}(t)(\mathcal{L} y)(t) .
\end{aligned}
$$

Summing up the above relation from $t_{0}$ to $m$, we get

$$
\begin{aligned}
S(y)(m+1)= & S(y)\left(t_{0}\right)+c \sum_{t=t_{0}}^{m} w(t)|y(t)|^{2}-\sum_{t=t_{0}}^{m} \bar{y}(t)(\mathcal{L} y)(t) \\
& +\sum_{t=t_{0}}^{m}\left(p_{0}(t)-c w(t)\right)|y(t)|^{2}+\sum_{j=1}^{n} \sum_{t=t_{0}}^{m} p_{j}(t)\left|\Delta^{j} y(t-j)\right|^{2} .
\end{aligned}
$$

For any $y \in \mathcal{D}$, it follows from Cauchy's inequality that the second and third items on the right side of $(2.15)$ converge. Since $p_{j}(t)(1 \leq j \leq n)$ and $p_{0}(t)-c w(t)$ are either non-negative for all $t \geq t_{0}$ or non-positive for all $t \geq t_{0}$, the last two items on the right side of (2.15) are monotonic. Therefore, $\lim _{t \rightarrow+\infty} S(y)(t)$ exists, finite or infinite. The proof is complete.

2.4. Asymptotic behaviors of solutions. At the end of this section, we consider asymptotic behaviors of solutions of equation (2.11).

Lemma 2.8. Assume that

$$
\sum_{t=0}^{+\infty} t^{2 n-1} w_{0}(t)<+\infty, \quad \sum_{t=0}^{+\infty} t^{2 n-1}\left|r_{j}(t)\right|<+\infty, \quad 0 \leq j \leq n-1 .
$$

Then, for any $\lambda \in \mathbb{C}$, equation (2.11) has a fundamental set of solutions $z_{j}(t)$, $1 \leq j \leq 2 n$, with the asymptotic form as $t \rightarrow+\infty$,

$$
z_{j}(t)=(1+o(1)) t^{j-1} .
$$


Proof. By a result of Coffman [6, p. 43], if the first condition in (2.16) holds and

$$
\sum_{t=0}^{+\infty} t^{2 n-1}\left|R_{j}(t)\right|<+\infty, \quad 0 \leq j \leq n-1,
$$

then for any $\lambda \in \mathbb{C}$, equation (2.10) has a fundamental set of solutions $z_{j}(t), 1 \leq$ $j \leq 2 n$, with the asymptotic form (2.17). By the relationship between $R_{j}(t)$ and $r_{j}(t)$, the second condition in (2.16) yields (2.18). Consequently, equation (2.11) has a fundamental set of solutions $z_{j}(t), 1 \leq j \leq 2 n$, with the asymptotic form (2.17). This completes the proof.

\section{MAin Results}

In this section, several results on the defect index of equation (1.1) and its equivalent forms are given. It is first shown that the defect index $d$ of equation (1.1) satisfies the inequality $n \leq d \leq 2 n$ and all values of $d$ in this range are realized. Next, several sufficient conditions of the limit point case for equations (2.9) and (1.1) are given. Finally, two criteria of the strong limit point case for equations (2.9) and (1.1) are established.

First, we give the following result based on asymptotic behaviors of solutions of equation (2.11):

Theorem 3.1. Consider equation (2.11). Assume that (2.16) holds. Then

(1) $d=n$ if $\sum_{t=0}^{+\infty} t^{2 n} w_{0}(t)=+\infty$;

(2) $d=n+s$ if $\sum_{t=0}^{+\infty} t^{2 n+2 s-2} w_{0}(t)<+\infty$ and $\sum_{t=0}^{+\infty} t^{2 n+2 s} w_{0}(t)=+\infty$ for some integer $1 \leq s \leq n-1$;

(3) $d=2 n$ if $\sum_{t=0}^{+\infty} t^{4 n-2} w_{0}(t)<+\infty$.

Proof. We prove only that (1) holds, and the others can be shown similarly. By Lemma 2.8, if (2.16) holds, then for any $\lambda \in \mathbb{C}$, equation (2.11) has a fundamental solution $z_{j}(t)=(1+o(1)) t^{j-1}, 1 \leq j \leq 2 n$. By the assumption in (1), it can be easily verified that $z_{j}(t), 1 \leq j \leq n$, belong to $l_{w_{0}}^{2}[0,+\infty)$, while $z_{j}(t), n+1 \leq j \leq 2 n$, do not belong to $l_{w_{0}}^{2}[0,+\infty)$. Moreover, any non-trivial linear combination of $z_{j}(t)$, $n+1 \leq j \leq 2 n$, is not in $l_{w_{0}}^{2}[0,+\infty)$. Hence, $d=n$. The proof is complete.

Example 3.2. Consider equation (2.11) in the special case: $w_{0}(t)=t^{\alpha}$, and $r_{j}(t)=$ $t^{\beta_{j}}$, where $\alpha, \beta_{j}, 0 \leq j \leq n-1$, are real numbers. It follows from Theorem 3.1 and Lemma 2.8 that if $\beta_{j}<-2 n, 1 \leq j \leq n-1$, then

$$
d= \begin{cases}n, & \text { if }-2 n-1 \leq \alpha<-2 n \\ n+s, & \text { if }-2 n-2 s-1 \leq \alpha<-2 n-2 s+1,1 \leq s \leq n-1 \\ 2 n, & \text { if } \alpha<-4 n+1\end{cases}
$$

This example shows that the defect index $d$ of equation (1.1) satisfies the inequality $n \leq d \leq 2 n$ and all values of $d$ in this range are realized. This parallels the above Glazman's result for differential equations.

Second, we give some sufficient conditions for the limit point case, that is, $d=n$. Similarly to the notation in Section 2, we introduce the following notation for 
equation (2.9):

$$
\begin{aligned}
\mathcal{L}_{1}(y)(t):= & \sum_{j=0}^{n}(-1)^{j} \Delta^{j}\left[q_{j}(t) \nabla^{j} y(t)\right], \\
{[x, y]_{1}(t):=} & \sum_{j=1}^{n}\left(\sum_{k=j}^{n}(-1)^{k-j} \Delta^{k-j}\left[q_{k}(t) \nabla^{k} \bar{y}(t)\right]\right) \Delta^{j-1} x(t-j) \\
& -\sum_{j=1}^{n} \Delta^{j-1} \bar{y}(t-j)\left(\sum_{k=j}^{n}(-1)^{k-j} \Delta^{k-j}\left[q_{k}(t) \nabla^{k} x(t)\right]\right), \\
S_{1}(y)(t):= & \sum_{j=1}^{n} \Delta^{j-1} \bar{y}(t-j)\left(\sum_{k=j}^{n}(-1)^{k-j} \Delta^{k-j}\left[q_{k}(t) \nabla^{k} y(t)\right]\right), \\
\mathcal{D}_{1}:=\{y \in & \left.l^{2}[0,+\infty): \mathcal{L}_{1} y \in l^{2}[0,+\infty)\right\} .
\end{aligned}
$$

Theorem 3.3. Assume that there exist a non-negative function $\sigma(t)$ defined on $I$, a constant $M>0$ and an integer $t_{0} \geq 0$ such that

$$
\sum_{t=0}^{+\infty} \sigma(t)=+\infty, \quad \sigma(t)\left|\Delta^{m} q_{s}(t)\right| \leq M, \quad t \geq t_{0},
$$

for all $1 \leq s \leq n$ and $0 \leq m \leq s-1$. Then equation (2.9) is in l.p.c. at $t=+\infty$.

Proof. By Lemma 2.4, it suffices to show that $\lim _{t \rightarrow+\infty}[x, y]_{1}(t)=0$ for all $x, y \in$ $\mathcal{D}_{1}$. By the discussion before Lemma $2.4, \lim _{t \rightarrow+\infty}[x, y]_{1}(t)$ exists and is finite for all $x, y \in \mathcal{D}_{1}$. Suppose that there exist $x, y \in \mathcal{D}_{1}$ such that $\lim _{t \rightarrow+\infty}[x, y]_{1}(t)=c \neq 0$. Then there exists $T_{0} \geq t_{0}$ such that $\left|[x, y]_{1}(t)\right| \geq|c| / 2>0$ for all $t \geq T_{0}$. We have from (2.1) and (2.2) that

$$
\begin{aligned}
& \sigma(t)\left|[x, y]_{1}(t)\right| \\
& \leq \sum_{j=1}^{n}\left(\left|\Delta^{j-1} x(t-j)\right| \sum_{k=j}^{n} \sum_{s=0}^{k-j} C_{k-j}^{s} \sigma(t)\left|\Delta^{s} q_{k}(t)\right|\left|\Delta^{2 k-j-s} \bar{y}(t+s-k)\right|\right) \\
& \quad+\sum_{j=1}^{n}\left(\left|\Delta^{j-1} \bar{y}(t-j)\right| \sum_{k=j}^{n} \sum_{s=0}^{k-j} C_{k-j}^{s} \sigma(t)\left|\Delta^{s} q_{k}(t)\right|\left|\Delta^{2 k-j-s} x(t+s-k)\right|\right),
\end{aligned}
$$

which, together with the second condition in (3.1) and Cauchy's inequality, implies that

$$
\begin{aligned}
\sigma(t)\left|[x, y]_{1}(t)\right| \leq & M \sum_{j=1}^{n} \sum_{k=j}^{n} \sum_{s=0}^{k-j} C_{k-j}^{s}\left(\left|\Delta^{j-1} x(t-j)\right|^{2}+\left|\Delta^{j-1} \bar{y}(t-j)\right|^{2}\right. \\
& \left.+\left|\Delta^{2 k-j-s} \bar{y}(t+s-k)\right|^{2}+\left|\Delta^{2 k-j-s} x(t+s-k)\right|^{2}\right) .
\end{aligned}
$$

Since $x, y \in \mathcal{D}_{1},\left|\Delta^{k} x\right|$ and $\left|\Delta^{k} \bar{y}\right|$ belong to $l^{2}[0,+\infty)$ for any $k \geq 1$. Hence,

$$
\sum_{t=T_{0}}^{+\infty} \sigma(t)\left|[x, y]_{1}(t)\right|<+\infty .
$$


On the other hand, it follows from the first condition in (3.1) that

$$
\sum_{t=T_{0}}^{+\infty} \sigma(t)\left|[y, z]_{1}(t)\right| \geq \frac{|c|}{2} \sum_{t=T_{0}}^{+\infty} \sigma(t)=+\infty .
$$

This is a contradiction. Therefore, $\lim _{t \rightarrow+\infty}[x, y]_{1}(t)=0$ holds for all $x, y \in \mathcal{D}_{1}$. By Lemma 2.4, equation (2.9) is in l.p.c. at $t=+\infty$.

Reversing the transformation from (1.1) to (2.9), we get the following result:

Theorem 3.4. Assume that there exist a non-negative function $\sigma(t)$ defined on $I$, a constant $M>0$ and an integer $t_{0} \geq n$ such that $\sigma(t)$ satisfies the first condition in (3.1) and

$$
\sigma(t)\left|\Delta^{m}\left[p_{s}(t+k+v) w^{-1 / 2}(t+v) w^{-1 / 2}(t-j+v)\right]\right| \leq M, \quad t \geq t_{0}
$$

for all $1 \leq s \leq n, 1 \leq j \leq s, 0 \leq k \leq s-j, 0 \leq v \leq j-1,0 \leq m \leq j-1-v$. Then equation (1.1) is in l.p.c. at $t=+\infty$.

Proof. Since equations (1.1) and (2.9) have the same limit case at $t=+\infty$ under the transformation (2.6), it suffices to show that the second condition in (3.1) holds by Theorem 3.3 .

From (2.4), (2.5) and (2.8) we have

$$
\begin{aligned}
q_{n}(t)= & p_{n}(t) w^{-1 / 2}(t) w^{-1 / 2}(t-n), \\
q_{j}(t)= & \sum_{s=j}^{n} \sum_{k=0}^{s-j} C_{s}^{k} C_{s}^{s-j-k} p_{s}(t+k) w^{-1 / 2}(t) w^{-1 / 2}(t-j) \\
& -\sum_{s=j+1}^{n} \sum_{k=0}^{s-j} C_{s}^{k} C_{s}^{s-j-k} q_{s}(t+k), \quad 1 \leq j \leq n-1 .
\end{aligned}
$$

When $s=n, j=n$ and $k=0$, it follows from (3.2) that

$$
\sigma(t)\left|\Delta^{m}\left[p_{n}(t+v) w^{-1 / 2}(t+v) w^{-1 / 2}(t-n+v)\right]\right| \leq M
$$

for all $0 \leq v \leq n-1,0 \leq m \leq n-1-v$, which together with (3.3) yields that

$$
\sigma(t)\left|\Delta^{m} q_{n}(t+v)\right| \leq M, \quad 0 \leq v \leq n-1,0 \leq m \leq n-1-v .
$$

In particular, setting $v=0$ in (3.5), one has

$$
\sigma(t)\left|\Delta^{m} q_{n}(t)\right| \leq M, \quad 0 \leq m \leq n-1 .
$$

When $s=n-1, j=n-1$ and $k=0$, it follows from (3.2) that

$$
\left|\sigma(t) \Delta^{m}\left[p_{n-1}(t+v) w^{-1 / 2}(t+v) w^{-1 / 2}(t-n+1+v)\right]\right| \leq M
$$

for all $0 \leq v \leq n-2,0 \leq m \leq n-2-v$. When $s=n, j=n-1$, (3.2) implies that

$$
\sigma(t)\left|\Delta^{m}\left[p_{n}(t+v+k) w^{-1 / 2}(t+v) w^{-1 / 2}(t-n+1+v)\right]\right| \leq M
$$

for all $0 \leq k \leq 1,0 \leq v \leq n-2,0 \leq m \leq n-2-v$. If $0 \leq v \leq n-2$ and $0 \leq k \leq 1$, then $0 \leq v+k \leq n-1$. So, from (3.5) we have

$$
\sigma(t)\left|\Delta^{m} q_{n}(t+k+v)\right| \leq M
$$

for all $0 \leq k \leq 1,0 \leq v \leq n-2,0 \leq m \leq n-2-v$. Hence, from (3.6), (3.7) and (3.8) we get

$$
\sigma(t)\left|\Delta^{m} q_{n-1}(t+v)\right| \leq M, \quad 0 \leq v \leq n-2,0 \leq m \leq n-2-v .
$$


Setting $v=0$ in (3.9), one has

$$
\sigma(t)\left|\Delta^{m} q_{n-1}(t)\right| \leq M, \quad 0 \leq m \leq n-2 .
$$

When $s=n-2, j=n-2$ and $k=0$, it follows from (3.2) that

$$
\sigma(t)\left|\Delta^{m}\left[p_{n-2}(t+v) w^{-1 / 2}(t+v) w^{-1 / 2}(t-n+2+v)\right]\right| \leq M
$$

for all $0 \leq v \leq n-3,0 \leq m \leq n-3-v$. When $s=n-1, j=n-2$, (3.2) implies that

$$
\sigma(t)\left|\Delta^{m}\left[p_{n-1}(t+v+k) w^{-1 / 2}(t+v) w^{-1 / 2}(t-n+2+v)\right]\right| \leq M
$$

for all $0 \leq k \leq 1,0 \leq v \leq n-3,0 \leq m \leq n-3-v$. When $s=n, j=n-2$, (3.2) yields that

$$
\sigma(t)\left|\Delta^{m}\left[p_{n}(t+v+k) w^{-1 / 2}(t+v) w^{-1 / 2}(t-n+2+v)\right]\right| \leq M
$$

for all $0 \leq k \leq 2,0 \leq v \leq n-3,0 \leq m \leq n-3-v$. If $0 \leq v \leq n-3$ and $0 \leq k \leq 1$, then $0 \leq v+k \leq n-2$. So from (3.9) we get

$$
\sigma(t)\left|\Delta^{m} q_{n-1}(t+k+v)\right| \leq M
$$

for all $0 \leq k \leq 1,0 \leq v \leq n-3,0 \leq m \leq n-3-v$. It is evident that $0 \leq v+k \leq n-1$ if $0 \leq v \leq n-3$ and $0 \leq k \leq 2$. So it follows from (3.5) that

$$
\sigma(t)\left|\Delta^{m} q_{n}(t+k+v)\right| \leq M
$$

for all $0 \leq k \leq 2,0 \leq v \leq n-3,0 \leq m \leq n-3-v$. Hence, from (3.10)-(3.14) we obtain

$$
\left|\sigma(t) \Delta^{m} q_{n-2}(t+v)\right| \leq M, \quad 0 \leq v \leq n-3,0 \leq m \leq n-3-v .
$$

Setting $v=0$ in (3.15), one has

$$
\sigma(t)\left|\Delta^{m} q_{n-2}(t)\right| \leq M, \quad 0 \leq m \leq n-3 .
$$

With a similar argument one can conclude that

$$
\sigma(t)\left|\Delta^{m} q_{s}(t+v)\right| \leq M
$$

for all $1 \leq s \leq n, 0 \leq v \leq s-1,0 \leq m \leq s-1-v$ by using (3.2). Therefore, the second condition in (3.1) holds by setting $v=0$ in (3.16). By Theorem 3.3, equation (2.9) is in l.p.c. at $t=+\infty$, which is equivalent to equation (1.1) being in l.p.c. at $t=+\infty$. The proof is complete.

Note that if there exist a constant $K>0$ and an integer $t_{0} \geq 0$ such that $|f(t)| \leq$ $K t$ for $t \geq t_{0}$, then there exists a constant $N>0$ such that $\left|\Delta^{m} f(t+v)\right| \leq N t$ for $t \geq t_{0}+1$ and $0 \leq m, v \leq n$. Thus the following result is a direct consequence of Theorem 3.4 by taking $\sigma(t)=t^{-1}$ :

Corollary 3.5. If there exist a constant $M>0$ and an integer $t_{0} \geq n$ such that

$$
\left|p_{s}(t+k)\right| \leq M t w^{1 / 2}(t) w^{1 / 2}(t-j), \quad t \geq t_{0},
$$

for all $1 \leq s \leq n, 1 \leq j \leq s$ and $0 \leq k \leq s-j$, then equation (1.1) is in l.p.c. at $t=+\infty$.

Now we give two remarks on Theorems 3.3 and 3.4.

Remark 3.6. Note that condition (3.1) of Theorem 3.3 does not impose a restriction on $q_{0}(t)$, and condition (3.2) of Theorem 3.4 does not place a restriction on $p_{0}(t)$. 
Remark 3.7. In the case of $n=1$, condition (3.2) is

$$
\sigma(t)\left|p_{1}(t) w^{-1 / 2}(t) w^{-1 / 2}(t-1)\right| \leq M, \quad t \geq t_{0} .
$$

It can be verified that the conditions of Theorem 3.4 in this case are equivalent to those of [2, Corollary 3.1] and [9, Theorem 10] as well as those of [13, Theorem 3.1] when the coefficients are real.

At the end of this section, two criteria of the strong limit point case for equations (2.9) and (1.1) are established, respectively.

Theorem 3.8. If $q_{j}(t), 0 \leq j \leq n$, satisfy all the conditions in Theorem 3.3 and Lemma 2.7, then equation (2.9) is in s.l.p.c. at $t=+\infty$.

Proof. Since $q_{j}(t), 0 \leq j \leq n$, satisfy the conditions in Lemma 2.7, we have that $\lim _{t \rightarrow+\infty} S_{1}(y)(t)$ exists and is finite or infinite for all $y \in \mathcal{D}_{1}$. With a similar argument to that used in the proof of Theorem 3.3, it follows that $\lim _{t \rightarrow+\infty} S_{1}(y)(t)=0$ for all $y \in \mathcal{D}_{1}$. By Lemma 2.6, (2.9) is in s.l.p.c. at $t=+\infty$. The proof is complete.

Theorem 3.9. If $p_{j}(t), 0 \leq j \leq n$, and $w(t)$ satisfy all the conditions in Theorem 3.4 and Lemma 2.7, then equation (1.1) is in s.l.p.c. at $t=+\infty$.

Proof. Since the proof is similar to that of Theorems 3.4 and 3.8, its details are omitted.

Remark 3.10. Sun and Shi [14] established several strong limit point criteria for the Hamiltonian system (1.3) with the weight function $W(t)=\operatorname{diag}\left\{W_{1}(t), W_{2}(t)\right\}$, where $W_{1}(t)$ and $W_{2}(t)$ are $n \times n$ Hermitian matrices, and it is required that $W_{1}(t)>0$. Although the $2 n$ th-order equation (1.1) can be converted into system (1.3) by (1.2), its weight function $W(t)=\operatorname{diag}\{w(t), 0, \ldots, 0\}$ from (1.4) does not satisfy the above condition in the case of $n \geq 2$. So, Theorems 3.8 and 3.9 are not included by their results in the case of $n \geq 2$.

\section{ACKNOWLEDGEMENT}

The authors thank the referee for valuable comments and suggestions.

\section{REFERENCES}

1. F. V. Atkinson, Discrete and Continuous Boundary Problems, Academic Press, Inc., New York, 1964. MR0176141 (31:416)

2. J. Chen and Y. Shi, The limit circle and limit point criteria for second-order linear difference equations, Comput. Math. Appl., 42(2001), 465-476. MR2060330 (2005d:39019)

3. S. L. Clark, A spectral analysis for self-adjoint operators generated by a class of second order difference equations, J. Math. Anal. Appl., 197(1996), 267-285. MR.1371289 (96m:47061)

4. S. L. Clark, F. Gesztesy, On Weyl-Titchmarsh theory for singular finite difference Hamiltonian systems, J. Comput. Appl. Math., 171(2004), 151-184. MR2077203 (2006i:39033)

5. E. A. Coddington, Extension theory of formally normal and symmetric subspaces, Mem. Amer. Math. Soc., 134(1973). MR0477855 (57:17357)

6. C. V. Coffman, Asymptotic behavior of solutions of ordinary difference equations, Trans. Amer. Math. Soc., 110(1964), 22-51. MR0156122 (27:6054)

7. A. Devinatz, The deficiency index problem for ordinary selfadjoint differential operators, Bull. Amer. Math. Soc., 79(1973), 1109-1127. MR0508316 (58:22742)

8. I. M. Glazman, On the theory of singular differential operators, Uspehi Math. Nauk 40(1950), 102-135; English translation in Amer. Math. Soc. Transl. Ser. 1, 4(1962), 331-372. MR0043389 (13:254d) 
9. D. B. Hinton, R. T. Lewis, Spectral analysis of second order difference equations, J. Math. Anal. Appl., 63(1978), 421-438. MR0611455 (58:29512)

10. A. Jirari, Second-order Sturm-Liouville difference equations and orthogonal polynomials, Mem. Amer. Math. Soc., 542(1995), 1-136. MR1227498 (95f:39003)

11. M. A. Naimark, Linear Differential Operators, Part II. Linear Differential Operators in Hilbert Space, Ungar Publ. Co., New York, 1968. MR0262880(41:7485)

12. Y. Shi, Weyl-Titchmarsh theory for a class of singular discrete linear Hamiltonian systems, Linear Algebra Appl., 416(2006), 452-519. MR2242741 (2007b:39034)

13. H. Sun and Y. Shi, Limit-point and limit-circle criteria for singular second-order linear difference equations with complex coefficients, Comput. Math. Appl., 52 (2006), 539-554. MR:2263520 (2007g:39005)

14. H. Sun and Y. Shi, Strong limit point criteria for a class of singular discrete linear Hamiltonian systems, J. Math. Anal. Appl., 336 (2007), 224-242. MR2348503 (2008j:39017)

15. J. Weidmann, Linear Operators in Hilbert Spaces, Springer-Verlag, New York, 1980. MR $566954(81 \mathrm{e}: 47001)$

School of Mathematics, Shandong University, Jinan, Shandong 250100, People's Republic of China - and - School of Statistics and Mathematics, Shandong Economic University, Jinan, Shandong 250014, People's Republic of China

E-mail address: rgjmaths@gmail.com

School of Mathematics, Shandong University, Jinan, Shandong 250100, People's Republic of China

E-mail address: ymshi@sdu.edu.cn 